\title{
Comunicação
}

[Communication]

\section{Freqüência de parasitos gastrintestinais em cães e gatos atendidos em hospital-escola veterinário da cidade de São Paulo}

\author{
[Frequency of gastrointestinal parasites in dogs and cats referred to a veterinary \\ school hospital in the city of São Paulo]
}

\author{
M.R. Funada, H.F.J. Pena, R.M. Soares, M. Amaku, S.M. Gennari* \\ Faculdade de Medicina Veterinária e Zootecnia-USP \\ Av. Prof. Orlando Marques de Paiva, 87 \\ 05508-270 - São Paulo, SP
}

As parasitoses gastrintestinais causadas por helmintos e protozoários estão entre as enfermidades mais comuns em cães e gatos, podendo ser especialmente graves em animais jovens ou imunocomprometidos (Bowman, 1995). Alguns desses parasitos como Ancylostoma spp., Toxocara spp., Giardia spp. e Cryptosporidium spp. podem provocar infecção em seres humanos (Robertson e Thompson, 2002).

Este estudo teve como objetivos determinar a freqüência dos diferentes gêneros de helmintos e protozoários gastrintestinais em amostras fecais de cães e gatos domiciliados, atendidos no Hospital Veterinário da Universidade de São Paulo, no período de janeiro de 2000 a dezembro de 2004, e identificar possíveis relações entre a presença dos parasitos e características dos hospedeiros como sexo, idade e raça.

As fezes foram examinadas pelos métodos de centrífugo-sedimentação em água-éter (Ferreira et al., 1962), centrífugo-flutuação em solução de sacarose $\left(\mathrm{d}=1,203 \mathrm{~g} / \mathrm{cm}^{3}\right)$ (Ogassawara et al., 1989) e flutuação em solução saturada de $\mathrm{NaCl}$ (Willis, 1921). O diagnóstico de Dipylidium caninum baseou-se na presença de proglotes nas fezes. Devido à impossibilidade de diagnóstico diferencial pelos métodos empregados, os oocistos de Hammondia heydorni ou Neospora caninum, quando encontrados nas fezes de cães, foram classificados como Hammondia-

Recebido em 18 de janeiro de 2006

Aceito em 31 de outubro de 2007

* Autor para correspondência (corresponding author)

E-mail: sgennari@usp.br
Neospora. Procedeu-se da mesma maneira para Hammondia hammondi ou Toxoplasma gondii, sendo classificados como HammondiaToxoplasma, quando presentes nas fezes de gatos.

Para fins de análise estatística, cães e gatos foram agrupados de acordo com a raça (com ou sem definição racial), idade ( $<$ ou $\geq 1$ ano) e sexo (macho ou fêmea). As diferenças entre as freqüências de parasitos entre cada agrupamento, foram analisadas pelo teste $\chi^{2}$, utilizando 0 programa Minitab 13 (Minitab Inc.). As diferenças foram consideradas estatisticamente significativas para $\mathrm{P} \leq 0,05$.

Das 1755 amostras fecais de cães examinadas, $486(27,7 \%)$ apresentaram um ou mais agentes parasitários. Ancylostoma spp. foi o parasito mais freqüente, seguido por Giardia spp., Cystoisospora spp., Toxocara canis e Cryptosporidium spp. (Tab. 1). Dentre os cães positivos, $386(79,4 \%)$ estavam infectados por um único parasito e $100(20,6 \%)$ por mais de um parasito. Giardia spp. ocorreu em $64 \%$ das amostras nas quais mais de um agente parasitário foi encontrado, sendo observada com maior freqüência em associações com Cryptosporidium spp. (16\%) e Cystoisospora spp. (14\%). Ainda, $51,7 \%$ eram machos e $48,3 \%$ fêmeas. Exceto para Trichuris vulpis, a freqüência de parasitos foi maior entre os machos para todos os gêneros de parasitos, mas diferença $(\mathrm{P} \leq 0,05)$ foi observada somente para Ancylostoma. 
Freqüência de parasitos gastrintestinais...

Tabela 1. Freqüência de ovos de helmintos, cistos e oocistos de protozoários segundo sexo e idade, em fezes de cães

\begin{tabular}{|c|c|c|c|c|c|c|c|c|c|c|}
\hline \multirow{3}{*}{ Parasitos } & \multirow{2}{*}{\multicolumn{2}{|c|}{$\begin{array}{c}\text { Total de amostras } \\
\text { positivas }\end{array}$}} & \multicolumn{4}{|c|}{ Sexo } & \multicolumn{4}{|c|}{ Idade } \\
\hline & & & \multicolumn{2}{|c|}{ Machos } & \multicolumn{2}{|c|}{ Fêmeas } & \multicolumn{2}{|c|}{$<1$ ano } & \multicolumn{2}{|c|}{$\geq 1$ ano } \\
\hline & $\mathrm{N}^{\mathrm{o}}$ & $(\%)$ & $\mathrm{N}^{\mathrm{o}}$ & $(\%)$ & $\mathrm{N}^{\mathrm{o}}$ & $(\%)$ & $\mathrm{N}^{\mathrm{o}}$ & $(\%)$ & $\mathrm{N}^{\mathrm{o}}$ & $(\%)$ \\
\hline Ancylostoma spp. & 223 & $(12,7)$ & 144 & $(64,6)^{* *}$ & 79 & $(35,4)^{* *}$ & 33 & $(14,8)^{*}$ & 190 & $(85,2)^{*}$ \\
\hline Giardia spp. & 149 & $(8,5)$ & 79 & $(53,0)$ & 70 & $(47,0)$ & 94 & $(63,1)^{*}$ & 55 & $(36,9)^{*}$ \\
\hline Cystoisospora spp. & 77 & $(4,4)$ & 42 & $(54,5)$ & 35 & $(45,4)$ & 58 & $(75,3)^{*}$ & 19 & $(24,7)^{*}$ \\
\hline Toxocara canis & 46 & $(2,6)$ & 30 & $(65,2)$ & 16 & $(34,8)$ & 33 & $(71,7)^{*}$ & 13 & $(28,3)^{*}$ \\
\hline Cryptosporidium spp. & 43 & $(2,4)$ & 22 & $(51,2)$ & 21 & $(48,8)$ & 28 & $(65,1)^{*}$ & 15 & $(34,9)^{*}$ \\
\hline Trichuris vulpis & 31 & $(1,8)$ & 15 & $(48,4)$ & 16 & $(51,6)$ & 6 & $(19,3)$ & 25 & $(80,7)$ \\
\hline Sarcocystis sp. & 25 & $(1,4)$ & 14 & $(56,0)$ & 11 & $(44,0)$ & 4 & $(16,0)$ & 21 & $(84,0)$ \\
\hline Hammondia-Neospora & 7 & $(0,4)$ & 5 & $(71,4)$ & 2 & $(28,6)$ & 2 & $(28,6)$ & 5 & $(71,4)$ \\
\hline Dipylidium sp. & 1 & $(0,06)$ & 0 & $(0,0)$ & 1 & $(100,0)$ & 0 & $(0,0)$ & 1 & $(100,0)$ \\
\hline Physaloptera sp. & 1 & $(0,06)$ & 0 & $(0,0)$ & 1 & $(100,0)$ & 0 & $(0,0)$ & 1 & $(100,0)$ \\
\hline
\end{tabular}

(**) Diferenças significativas entre machos e fêmeas $(\mathrm{P} \leq 0,05)$

(*) Diferenças significativas entre cães com idade $<1$ ano $\mathrm{e} \geq 1$ ano $(\mathrm{P} \leq 0,05)$.

A idade teve considerável influência $(\mathrm{P} \leq 0,05)$ sobre a freqüência da maioria dos parasitos. Ancylostoma spp. esteve associado a cães com idade acima de um ano, semelhante aos resultados encontrados por Gennari et al. (2001) e Eguía-Aguilar et al. (2005). Os gêneros Giardia, Cryptosporidium, Cystoisospora e Toxocara estavam presentes com maior freqüência em cães com idade inferior a um ano $(\mathrm{P} \leq 0,05)$, assim como relatado por Gennari et al. (2001). Visco et al. (1977) e Ramírez-Barrios et al. (2004) também verificaram prevalência mais alta de Cystoisospora e Toxocara em filhotes, quando comparadas à de adultos.

Não houve diferença significativa $(\mathrm{P}>0,05)$ entre as freqüências de cães infectados com e sem definição racial, e $78,7 \%$ deles eram de raças definidas. Dentre as 10 raças mais comuns, as com maiores freqüências de parasitos foram Husky Siberiano (42,9\%), Labrador (40,3\%) e Rottweiller (37,9\%). Dachshund, Yorkshire e Poodle foram as raças menos afetadas, com freqüências de 10,2\%, 13,6\% e $16,4 \%$, respectivamente. Cães domiciliados de grande porte parecem sofrer maior exposição aos parasitas. Pode-se supor que a maioria deles viva em casas, onde é mais freqüente a presença de solos permeáveis, favoráveis à presença das formas infectantes. $\mathrm{O}$ maior acesso à rua também aumentaria os riscos de infecção, principalmente por meio do contato com solos contaminados.

Em $103(31,5 \%)$ dos 327 gatos foi observada a presença de pelo menos um gênero de parasito. $\mathrm{O}$ protozoário Cryptosporidium spp. foi o mais freqüente, seguido por Giardia spp., Cystoisospora spp. e Toxocara cati (Tab. 2). Maior freqüência de protozoários em relação aos helmintos também foi encontrada por McGlade et al. (2003), na Austrália. A freqüência de parasitos entre os gatos foi semelhante $(\mathrm{P}>0,05)$ em relação ao sexo, como observado por Visco et al. (1978). Quanto à idade, $81,1 \%$ dos gatos com Cryptosporidium spp. e 66,7\% com Cystoisospora spp. tinham menos de um ano $(\mathrm{P} \leq 0,05)$.

Tabela 2. Freqüência de ovos de helmintos, cistos e oocistos de protozoários segundo sexo e idade, em fezes de 327 gatos

\begin{tabular}{|c|c|c|c|c|c|c|c|c|c|c|}
\hline \multirow{3}{*}{ Parasitos } & \multirow{2}{*}{\multicolumn{2}{|c|}{$\begin{array}{c}\text { Total de amostras } \\
\text { positivas }\end{array}$}} & \multicolumn{4}{|c|}{ Sexo } & \multicolumn{4}{|c|}{ Idade } \\
\hline & & & \multicolumn{2}{|c|}{ Macho } & \multicolumn{2}{|c|}{ Fêmea } & \multicolumn{2}{|c|}{$<1$ ano } & \multicolumn{2}{|c|}{$\geq 1$ ano } \\
\hline & $\mathrm{N}^{\mathrm{o}}$ & $(\%)$ & $\mathrm{N}^{\mathrm{o}}$ & $(\%)$ & $\mathrm{N}^{\mathrm{o}}$ & $(\%)$ & $\mathrm{N}^{\mathrm{o}}$ & $(\%)$ & $\mathrm{N}^{\mathrm{o}}$ & $(\%)$ \\
\hline Cryptosporidium spp. & 37 & $(11,3)$ & 20 & $(54,0)$ & 17 & $(45,9)$ & 30 & $(81,1)^{*}$ & 7 & $(18,9)^{*}$ \\
\hline Giardia spp. & 27 & $(8,3)$ & 17 & $(63,0)$ & 10 & $(37,0)$ & 16 & $(59,3)$ & 11 & $(40,7)$ \\
\hline Cystoisospora spp. & 27 & $(8,3)$ & 15 & $(55,6)$ & 12 & $(44,4)$ & 18 & $(66,7)^{*}$ & 9 & $(33,3) *$ \\
\hline Toxocara cati & 20 & $(6,1)$ & 10 & $(50,0)$ & 10 & $(50,0)$ & 11 & $(55,0)$ & 9 & $(45,0)$ \\
\hline Ancylostoma spp. & 7 & $(2,1)$ & 5 & $(71,4)$ & 2 & $(28,6)$ & 1 & $(14,3)$ & 6 & $(85,7)$ \\
\hline Sarcocystis sp. & 6 & $(1,8)$ & 1 & $(16,7)$ & 5 & $(83,3)$ & 2 & $(33,3)$ & 4 & $(66,7)$ \\
\hline $\begin{array}{l}\text { Hammondia- } \\
\text { Toxoplasma }\end{array}$ & 2 & $(0,6)$ & 0 & $(0,0)$ & 2 & $(100,0)$ & 2 & $(100,0)$ & 0 & $(0,0)$ \\
\hline Dipylidium sp. & 1 & $(0,3)$ & 0 & $(0,0)$ & 1 & $(100,0)$ & 0 & $(0,0)$ & 1 & $(100,0)$ \\
\hline Platynosomun spp. & 1 & $(0,3)$ & 0 & $(0,0)$ & 1 & $(100,0)$ & 0 & $(0,0)$ & 1 & $(100,0)$ \\
\hline
\end{tabular}


O número de gatos sem raça definida $(77,1 \%)$ predominou sobre os com raça definida $(22,9 \%)$. Não houve diferença $(\mathrm{P}>0,05)$ entre as freqüências de gatos infectados com ou sem raça definida.

Os resultados deste estudo mostram que os parasitos mais freqüentes tanto nos cães (Ancylostoma spp. e Giardia spp.) quanto nos gatos (Cryptosporidium spp. e Giardia spp.) são agentes de doenças zoonóticas e, portanto, de grande relevância na saúde pública. Deve-se ressaltar a importância do controle periódico das parasitoses gastrintestinais em cães e gatos, com base no correto diagnóstico e uso adequado de antiparasitários, bem como o emprego de medidas de preventivas.

Palavras-chave: cães, gatos, helmintos, protozoários, freqüência

\begin{abstract}
Fecal samples from 1755 dogs and 327 cats were examined for the presence of helminths and protozoan forms. From the total samples, 486 (27.7\%) dogs and 103 (31.5\%) cats presented at least one parasite. The main genus of parasite in dogs were Ancylostoma (12.7\%), Giardia (8.5\%), Cystoisosopora (4.4\%), Toxocara (2.6\%), and Cryptosporidium (2.4\%). The ocurrence of Ancylostoma was associated to male dogs, older than one year, while Giardia, Cryptosporidium, Cystoisospora and Toxocara were associated to dogs younger than one year $(P \leq 0.05)$. Among cats, the most frequent parasites were Cryptosporidium (11.3\%), Giardia (8.3\%), Cystoisosopora (8.3\%), Toxocara (6.1\%), and Ancylostoma (2.1\%). Cryptosporidium and Cystoisosopora were more prevalent in cats younger than one year $(P \leq 0.05)$.
\end{abstract}

Keywords: dog, cat, helminths, protozoa, frequency

\section{REFERÊNCIAS BIBLIOGRÁFICAS}

BOWMAN, D.D. Georgis' parasitology for veterinarians. 6.ed. Philadelphia: Saunders, 1995. 430 p.

EGUÍA-AGUILAR， P.; CRUZ-REYES， A.; MARTÍNEZ-MAYA, J.J. Ecological analysis and description of the intestinal helminths present in dogs in Mexico City. Vet. Parasitol., v.127, p.139-146, 2005.

FERREIRA, L.F.; MORTEO, R.E.; SILVA, J.R. Padronização de técnicas para exame parasitológico das fezes. J. Brasil. Med., v.6, p.241-257, 1962.

GENNARI, S.M.; PENA, H.F.J.; BLASQUES, L.S. Freqüência de ocorrência de parasitos gastrintestinais em amostras de fezes de cães e gatos da cidade de São Paulo. Vet News, v.52, p.10-12, 2001.

McGLADE, T.R.; ROBERTSON, I.D.; ELLIOT, A.D. et al. Gastrointestinal parasites of domestic cats in Perth, Western Australia. Vet. Parasitol., v.117, p.251-262, 2003.

OGASSAWARA, S.; CASTRO, J.M.; KASAI, N. et al. Cryptosporidium tipo C. muris em bovinos do Estado de São Paulo. In: SEMINÁRIO BRASILEIRO DE PARASITOLOGIA VETERINÁRIA, 6., 1989, Bagé. Anais... Bagé, 1989. p.123.

RAMÍREZ-BARRIOS, R.A.; BARBOZAMENA, G.; MUÑOZ, J. et al. Prevalence of intestinal parasites in dogs under veterinary care in Maracaibo, Venezuela. Vet. Parasitol., v.121, p.11-20, 2004.

ROBERTSON, I.D.; THOMPSON, R.C. Enteric parasitic zoonoses of domesticated dogs and cats. Microb. Infect., v.4, p.867-873, 2002.

VISCO, R.J.; CORWIN, R.M.; SELBY, L.A. Effect of age and sex on the prevalence of intestinal parasitism in dogs. J. Am. Vet. Med. Assoc., v.170, p.835-837, 1977.

VISCO, R.J.; CORWIN, R.M.; SELBY, L.A. Effect of age and sex on the prevalence of intestinal parasitism in cats. J. Am. Vet. Med. Assoc., v.172, p.797-800, 1978.

WILLIS, H.H. A simple levitation method for the detection of hookworm ova. Med. J. Aust., v.2, p.375-376, 1921. 